\title{
An interpretation of two x-ray studies of vanadium sesquioxide $\left(\mathrm{V}_{2} \mathrm{O}_{3}\right)$
}

\author{
S. W. Lovesey, D. S. Sivia and K. S. Knight \\ ISIS Facility, Rutherford Appleton Laboratory, \\ Oxfordshire OX11 0QX, England, UK
}

\begin{abstract}
Recent studies of $\mathrm{V}_{2} \mathrm{O}_{3}$ include experiments performed with x-rays, and exploit atomic resonances of $\mathrm{V}$ ions. In one case the $\mathrm{K}$-edge resonance has been used to enhance Bragg intensities, and in a second experiment the absorption signal has been monitored at $\mathrm{L}_{2}$ and $\mathrm{L}_{3}$ edges. Data collected on magnetically order $\mathrm{V}_{2} \mathrm{O}_{3}$ is successfully interpreted on the basis of an atomic model, and the established chemical and magnetic structures. The extensive diffraction data gathered in azimuthal-angle scans at charge-forbidden reflections is found to infer the $\mathrm{V}$ ion wavefunction, which in turn permits estimates to be made of atomic quantities not measured by diffraction, and including the absorption signal.
\end{abstract}

S. W. Lovesey, ISIS Facility, Rutherford Appleton Laboratory, Chilton, Didcot, Oxon OX11 0QX, UK, Fax No.: +44 (0) 123544 5642, E-mail: s.w.lovesey@rl.ac.uk. 


\section{Introduction}

Vanadium sesquioxide $\left(\mathrm{V}_{2} \mathrm{O}_{3}\right)$ displays a number of electronic, magnetic and structural properties that are challenging to interpret and explain [1-7]. At room temperature $\mathrm{V}_{2} \mathrm{O}_{3}$ has the corundum structure with space group $R \overline{3} c$, and it is metallic and paramagnetic. (It is unique among the iso-structural sesquioxides with respect to its small $a / c$ ratio and metallic conductivity.) On reducing the temperature the corundum structure distorts to a monoclinic structure with space group $I 2 / a$. The structural transition in the temperature range $150-160 \mathrm{~K}$ is strongly first-order and ferroelastic. Accompanying the transition are a change from metallic to insulating behaviour and the onset of antiferromagnetic order [3]. The metal-insulator transition is viewed as a classic Mott transition.

Here we report interpretations of two recent x-ray experiments. First, resonant x-ray Bragg diffraction at the $\mathrm{V}$ K-edge has revealed new magnetic features not seen in magnetic neutron diffraction $[8-10]$. One feature is a selection rule on the intensity of Bragg diffraction that allows electric dipole (E1) contributions at reflections indexed by Miller indices $h k l$ which satisfy $h+k+l$ an odd integer and even $h$. This class of reflections has been used to establish the configuration of $\mathrm{V}$ magnetic moments by neutron diffraction [3]. Reflections indexed by $h+k+l$ an odd integer and odd $h$ are due to scattering enhanced by an electric quadrupole (E2) event and E1 scattering is forbidden. This class of reflection is the magnetic analogue of Templeton and Templeton scattering $[11,12]$ by anisotropic charge distributions. It remains to be seen whether current neutron diffractometers are capable of observing scattering at spacegroup forbidden and odd $h$ reflections.

The second type of experiment of interest is $\mathrm{x}$-ray absorption performed at the $\mathrm{V} \mathrm{L}_{2}$ and $\mathrm{L}_{3}(2 \mathrm{p} \rightarrow 3 \mathrm{~d})$ edges [13]. We show that the integrated signal is proportional to the $\mathrm{V}$ quadrupole moment. The signal is estimated, on the basis of the successful interpretation of resonant diffraction data, and the value is consistent with observation.

The resonance enhanced intensity is related to properties of the valence shell that accommodates the photo-ejected core electron in the intermediate state of the elastic scattering process. Absorption at the K-edge and an electric-dipole event (E1) gives access to valence states with atomic p-like character, and an electric quadrupole event (E2) at the same edge gives access to d-like states $(1 \mathrm{~s} \rightarrow 3 \mathrm{~d})$. We analyse in terms of a pure E2 event diffraction data collected with the primary energy tuned to a feature in the pre-edge 
(5.464 keV and $\lambda=2.27 \AA$ ) of the vanadium K-shell. The associated Bragg intensity is therefore attributed directly to properties of the $3 \mathrm{~d}$-shell of a vanadium ion. As a function of the primary energy, the observed intensity is adequately described by a single oscillator. In this instance, we can confidently ascribe the intensity to orbital degrees of freedom [14] and spin degrees of freedom are not explicitly contributing. (This statement has been confirmed by two studies of diffraction by $\mathrm{NiO}$ with enhancement from an E2 event at the K-edge of the Ni ion [15].) For magnetically ordered $\mathrm{V}_{2} \mathrm{O}_{3}$ we demonstrate that charge-forbidden reflections are due to the orbital magnetization (specifically, the amplitude is the mean value of spherical tensors of odd rank that are odd with respect to time reversal). The intensity must decrease on warming the sample and vanish with the loss of magnetic long-range order.

There are two main ingredients in the calculated structure factors. First, knowledge of the chemical and magnetic structures. Information on the crystal structure is based on observations reported by Dernier and Marezio [2]. The second ingredient is a model for the resonant part of the scattering length. We employ an atomic model which should be fully adequate for a one-component intensity profile like the ones observed in diffraction at the Kedge of $\mathrm{V}_{2} \mathrm{O}_{3}$ [8 - 10]. The same atomic model should provide a reliable estimate of the integrated absorption profile $[13,16]$.

\section{Chemical and magnetic structures}

At room temperature vanadium sesquioxide has a trigonal (corundum) structure with space group $167(R \overline{3} c)$. Lowering the temperature of the material induces distortions which include tilting of the trigonal (hexagonal-c) axis and reduction of the point-group symmetry of sites occupied by vanadium ions from $3\left(C_{3}\right)$ to that of no symmetry.

The space group of the low temperature monoclinic structure is number 15(I2/a) in which vanadium ions occupy sites $8(f)$. This is a body-centred cell and Bragg wavevectors $\tau_{m}(h k l)$ for charge reflections have the necessary condition $h+k+l$ an even integer. (Miller indices $h, k$ and $l$ are integers.) In neutron diffraction patterns, magnetic reflections are indexed by $h+k+l$ odd [3]. The antiferromagnetic configuration of vanadium magnetic moments, displayed in Fig. 1, consists of sheets of moments with ferromagnetic alignment within $(010)_{m}$ layers, or hexagonal (110) layers, and moment reversal between adjacent layers. The moments 
are orientated along some easy-axis in these layers, and we take $\phi$ as the canting angle with respect to the trigonal axis.

The trigonal basis vectors are $\mathbf{a}_{h}=a(1,0,0), \mathbf{b}_{h}=a(-1 / 2,1 / 2 \sqrt{3}, 0)$ and $\mathbf{c}_{h}=c(0,0,1)$ and the volume of the unit cell $=a^{2} c \sqrt{3} / 2$. Following Dernier and Marezio [2] in the use of an $I$ - centred cell, from these vectors we generate monoclinic basis vectors $\mathbf{a}_{m}=\left(0, \frac{1}{\sqrt{3}} 2 a, c / 3\right)$, $\mathbf{b}_{m}=\mathbf{a}_{h}$ and $\mathbf{c}_{m}=\left(0, \frac{1}{\sqrt{3}} a,-c / 3\right)$, and the volume of the cell $=a^{2} c / \sqrt{3}$. The corresponding Bragg wavevector $\tau_{m}(h k l) \equiv(h k l)_{m}$ is,

$$
\boldsymbol{\tau}_{m}(h k l)=\frac{1}{a}\left(k, \frac{1}{\sqrt{3}}(h+l), \frac{a}{c}(h-2 l)\right)
$$

We note that $(l 0 \bar{l})_{m}$ is parallel to $\mathbf{c}_{h}$ and $(2 l k l)_{m}$ is normal to $\mathbf{c}_{h}$.

Referring to Fig. 1, the position coordinates of vanadium ions labelled (1) and (5) are $(x, y, z)$ and $(-x,-y,-z)$, respectively, with $x=0.3439, y=0.0012$ and $z=0.2993$ [2].

The positions of the pair (2) and (6) are related by a body-centre translation to the pair (1) and (5). The position coordinates of (3) and (7) are $(1 / 2-x, y,-z)$ and $(1 / 2+x,-y, z)$, respectively, and the pairs (4) and (8), and (3) and (7) are related by the body-centre translation. The bodycentre translation $(1 / 2,1 / 2,1 / 2)_{m}=\frac{a}{2}(1, \sqrt{3}, 0)$ and $(1 / 2,1 / 2,1 / 2)_{m} \cdot \tau_{m}(h k l)=1 / 2(h+k+l)$. It is convenient to define an angle $v=2 \pi(x, y, z)_{m} \cdot \tau_{m}(h k l)=2 \pi(x h+y k+z l)$ and we take $y k=0$.

\section{The structure factor for magnetically ordered $\mathrm{V}_{2} \mathrm{O}_{3}$}

As a function of the energy of the primary x-rays, the observed intensity of diffraction from $\mathrm{V}_{2} \mathrm{O}_{3}$ which is enhanced by electric-quadrupole (E2) resonance has a simple shape $[8,9]$ which is adequately described by a single oscillator. In this instance, the integrated intensity can be calculated without approximation [14], and for a pure E2 absorption event 1s $\rightarrow 3 \mathrm{~d}$ the intensity is found to be proportional to the orbital moments of the $3 \mathrm{~d}$ valence shell [16,17]. The orbital moments are represented by atomic tensors $\left\langle\mathbf{T}^{(K)}\right\rangle$ of rank $K=0,1,2,3$ and 4. (For electric-dipole absorption the maximum $K$ is 2.) In the structure factor, which is a scalar quantity, the atomic tensors appear in a scalar product with a tensor, denoted by $\mathbf{H}^{(K)}$, that describes the conditions of the primary and diffracted beams of X-rays. It is convenient in the ensuing calculation to consider the quantity, 


$$
\Psi_{Q}^{(K)}=\sum_{\mathbf{d}}<T_{Q}^{(K)}(\mathbf{d})>\exp (\mathrm{i} \boldsymbol{\tau} \cdot \mathbf{d})
$$

where $-K \leq Q \leq K$, $\mathbf{d}$ defines the position of a resonant vanadium ion in the unit cell and $\tau$ is the Bragg wavevector for the reflection in question. With this notation the structure factor is $[14,17]$

$$
F(\boldsymbol{\tau})=\sum_{K}(2 K+1)^{1 / 2} \mathbf{H}^{(K)} \cdot \boldsymbol{\Psi}^{(K)}=\sum_{K, Q}(2 K+1)^{1 / 2}(-1)^{Q} H_{-Q}^{(K)} \Psi_{Q}^{(K)}
$$

The components $H_{Q}^{(K)}$ for unrotated $\left(\sigma^{\prime} \sigma\right)$ and rotated $\left(\pi^{\prime} \sigma\right)$ radiation are tabulated [17]. The tensor $\mathbf{H}^{(K)}$ and $\Psi^{(K)}$ are evaluated in the coordinate system that defines the experimental geometry.

We find it convenient initially to consider $\Psi_{Q}^{(K)}$ with respect to a second set of orthogonal axes $(x, y, z)$ that include $\mathbf{a}_{h}=(a, 0,0)=\mathbf{b}_{m}$ and $\mathbf{c}_{h}=(0,0, c)$. Thereafter we apply to $\Psi_{Q}^{(K)}$ rotations that describe the orientation of the crystal in the coordinates of the experimental geometry that apply to $\mathbf{H}^{(K)}$.

The eight resonant vanadium ions are labelled according to the scheme shown in Fig 1. In structure number 15 the chemical environments of ions in the pairs (1) \& (2), (5) \& (6), (7) \& (8) and (3) \& (4) are identical. Moreover, the axes of quantization of ions in a pair are oppositely aligned and, in consequence, their atomic tensors differ by a phase factor $(-1)^{K}$ [17]. One finds for (3.1) the result,

$$
\begin{aligned}
\Psi_{Q}^{(K)}= & \left\{1-(-1)^{K}\right\}\left\{\mathrm{e}^{\mathrm{iv}}\left[<T_{Q}^{(K)}(1)>+(-1)^{h}<T_{Q}^{(K)}(7)>\right]\right. \\
& \left.+\mathrm{e}^{-\mathrm{iv}}\left[<T_{Q}^{(K)}(5)>+(-1)^{h}<T_{Q}^{(K)}(3)>\right]\right\} .
\end{aligned}
$$

Evidently, the structure factor for charge-forbidden reflections $(h+k+l$ odd $)$ is constructed from atomic tensors with $K=1$ and $K=3$. The odd-rank tensors are purely magnetic and $\left\langle\mathbf{T}^{(1)}\right\rangle=\langle\mathbf{L}\rangle / \sqrt{30}$ and $\left\langle\mathbf{T}^{(3)}\right\rangle=\langle\Lambda\rangle / 3 \sqrt{70}$ where $\langle\mathbf{L}\rangle$ and $\langle\Lambda\rangle$ are, respectively, the orbital 
magnetic moment and the orbital octupole moment of the $3 \mathrm{~d}$-valence shell of a resonant vanadium ion.

Vanadium ions at sites (1) \& (5) are related by inversion. The atomic tensor, and $\mathbf{L}$, is unchanged by inversion and thus $<T_{Q}^{(K)}(1)>=<T_{Q}^{(K)}(5)>$. A similar relation holds for (3) \& (7). Using this information in (3.3), and taking $K$ to be an odd integer, one finds,

$$
\Psi_{Q}^{(K)}=4 \cos (v)\left\{<T_{Q}^{(K)}(1)>+(-1)^{h}<T_{Q}^{(K)}(7)>\right\} .
$$

Ions at sites (1) \& (7) are related by an a-glide that includes reflection in the $\mathbf{a}_{m}-\mathbf{c}_{m}$ plane, which is normal to $\mathbf{a}_{h}=\mathbf{b}_{m}$. Reflection in the $\mathbf{a}_{m}-\mathbf{c}_{m}$ plane amounts to the change $x \rightarrow-x$, and with it $\left\langle T_{Q}^{(K)}>\rightarrow(-1)^{K}<T_{-Q}^{(K)}>\right.$. For $K=1 \quad\left(L_{x}, L_{y}, L_{z}\right) \rightarrow\left(L_{x},-L_{y},-L_{z}\right)$, so that reflection in the $\mathbf{a}_{m}-\mathbf{c}_{m}$ plane is accompanied by a change in the orientation of the orbital magnetization. The configuration of moments determined by Moon [3] is preserved if the polarity of the local field is now reversed, leading to $\left(L_{x},-L_{y},-L_{z}\right) \rightarrow\left(-L_{x}, L_{y}, L_{z}\right)$, for according to Moon $\left\langle L_{x}\right\rangle=0$ and the moments are confined to the $\mathbf{a}_{m}-\mathbf{c}_{m}$ plane. Reversing the polarity of the local field, which is sometimes described as time-reversal, introduces in the atomic tensor a phase factor $(-1)^{K}$. The appropriate relation between tensors at sites (1) \& (7) is seen to be the union of reflection in the $\mathbf{a}_{m}-\mathbf{c}_{m}$ plane and time-reversal, and our discussion leads to the result $\left\langle T_{Q}^{(K)}(7)\right\rangle$ $=\left\langle T_{-Q}^{(K)}(1)\right\rangle$; for simplicity of notation, we hereafter write $\left\langle T_{Q}^{(K)}\right\rangle=<T_{Q}^{(K)}(1)>$.

On using in (3.4) our result for the atomic tensor for the vanadium ion at site (7) we arrive at,

$$
\Psi_{Q}^{(K)}=4 \cos (v)\left\{<T_{Q}^{(K)}>+(-1)^{h}<T_{-Q}^{(K)}>\right\}
$$

The result (3.5) applies for $h+k+l$ odd (a charge-forbidden reflection) and $K$ odd (purely magnetic diffraction), and it is the basis of the subsequent interpretation of azimuthal-angle scans performed on magnetically ordered $\mathrm{V}_{2} \mathrm{O}_{3}$. According to (3.5), reflections with $h$ even are described by $\Psi_{Q}^{(K)}=\Psi_{-Q}^{(K)}$, whereas for $h$ odd $\Psi_{Q}^{(K)}$ is an odd function of $Q$ and $\Psi_{0}^{(K)}=0$. 
In the monoclinic structure, sites occupied by the vanadium ions possess no spatial symmetry and one can choose any set of axes for the associated atomic tensors. When proceeding from the corundum structure to the monoclinic one the average vanadium-oxygen distance remains essentially constant [3]. This aspect of the structural transition, and others mentioned in section 2, indicate that in the monoclinic structure the potential field experienced by a vanadium ion is principally referred to the trigonal axis, $\mathbf{c}_{h}$, and the field is almost subject to the requirement of a three-fold axis of rotation. An atomic tensor invariant with respect to a three-fold rotation is zero unless the projection index $Q$ has the values 0 or \pm 3 . Additions to the potential field, which reduce the symmetry from $\mathrm{C}_{3}$ to one of no spatial symmetry, lift the restriction on the allowed values of $Q$.

With $h$ odd $\Psi_{0}^{(K)}=0$ and we are led to consider $\Psi_{ \pm 1}^{(1)}$, and $\Psi_{Q}^{(3)}$ evaluated for $Q= \pm 1, \pm$ 2, and \pm 3 . Concerning $\Psi_{ \pm 1}^{(1)}$ we know that $\left\langle L_{x}\right\rangle=\left\langle L_{-1}-L_{+1}\right\rangle / \sqrt{2}=0$, because the magnetic moment is confined to the $\mathbf{a}_{m}-\mathbf{c}_{m}$ plane, and from this condition it follows that $\Psi_{ \pm 1}^{(1)}=0$. For diffraction enhanced by an E1 absorption event, which entails atomic tensors of rank up to $K=2$, we reach the conclusion that when both $h+k+l$ and $h$ are odd the E1 structure factor is zero. This selection rule is consistent with the experimental observations [8, 9].

\section{Azimuthal-angle scans}

Intensities calculated from (3.2) and (3.5) have been confronted with data gathered in azimuthal-angle scans, in which the crystal is rotated about $\tau_{m}(h k l)$. Typical results of a fit to data are shown in Fig. 2. Additional results and full details about the calculation of intensities in azimuthal-angle scans are found in reference [18].

From fitting to the x-ray data we find the canting angle $\phi \sim 70^{\circ}$, and this value is consistent with neutron diffraction data [3]. Using all available data and our model we can infer the wavefunction of a $\mathrm{V}$ ion in magnetically ordered $\mathrm{V}_{2} \mathrm{O}_{3}$ [19]. We have used this information to estimate the $\mathrm{V}$ quadrupole moment, among other quantities, which determines the dichroic signal at $\mathrm{L}_{2}$ and $\mathrm{L}_{3}$ absorption edges. This topic is taken up in the next section. 


\section{Absorption at $\mathrm{V} \mathrm{L}_{2}$ and $\mathrm{L}_{3}$ edges.}

Absorption is proportional to the imaginary part of the scattering length evaluated for forward scattering, i.e. $h=k=l=0$. Following the steps in section 3 , for this condition on Miller indices, shows that the appropriate structure factor can be different from zero for even $K$, and one finds,

$$
\Psi_{Q}^{(K)}=4\left\{<T_{Q}^{(K)}>+<T_{-Q}^{(K)}>\right\}
$$

This result is used in a theory of E1 absorption events [16] to provide integrated signals at the $\mathrm{L}_{2}$ and $\mathrm{L}_{3}$ edges.

Park et al. [13] used a sample with the face cleaved normal to $(1,0,2)_{\mathrm{h}}$, which is parallel to $\mathbf{n}=(\sqrt{3} / 2,1 / 2, a \sqrt{3} / c)_{m}$. With $a / c=0.354$ the angle enclosed by $\mathbf{n}$ and $\mathbf{c}_{\mathrm{h}}$ is $58.5^{\circ}$. Two settings are used in the experiment, which leave the primary $\sigma$-polarization parallel and perpendicular to $\mathbf{c}_{\mathrm{h}}$, to a good approximation. The absorption signals are related to the following atomic quantities;

(a) $\sigma / / \mathbf{c}_{\mathrm{h}}$

$$
\sqrt{6}<T_{0}^{(2)}>+\cos (2 \gamma)\left[<T_{+2}^{(2)}>+<T_{-2}^{(2)}>\right]
$$

(b) $\sigma \perp \mathbf{c}_{\mathrm{h}}$

$$
2 \cos (2 \gamma)\left[<T_{+2}^{(2)}>+<T_{-2}^{(2)}>\right]
$$

The angle $\gamma$ is determined by $\mathbf{n}$ and it turns out to be independent of $a / c$, namely, cos $(2 \gamma)=1 / 2$. The atomic tensor $\left\langle\mathbf{T}^{(2)}\right\rangle[16]$ is related to the $\mathrm{V}$ quadrupole moment $\langle\mathbf{Q}\rangle$ and two other ranktwo tensors denoted by $\langle\mathbf{P}\rangle$ and $\langle\mathbf{R}\rangle$,

$$
<\mathbf{T}^{(2)}>\alpha(2 \bar{J}+1)<\mathbf{Q}> \pm \frac{12}{5}[<\mathbf{P}>+<\mathbf{R}>]
$$


where the upper choice of sign is for the $\mathrm{L}_{3}(\bar{J}=3 / 2)$ edge and the lower sign is for the $\mathrm{L}_{2}(\bar{J}=1 / 2)$ edge. The total signal is seen to be proportional to $\langle\mathbf{Q}\rangle$ [20], and in our notation it is positive for an isotropic distribution of holes in the valence shell. The difference in the signal for the two settings (a) and (b) of $\sigma$-polarization is zero for a spherical charge distribution, and we find it has the value,

$$
\frac{2}{15}<2 \mathrm{p}|R| 3 \mathrm{~d}>^{2}\left\{<Q_{\mathrm{zz}}>-\frac{1}{6}<Q_{\mathrm{xx}}-Q_{\mathrm{yy}}>\right\} .
$$

The coordinates $(x, y, z)$ are defined in section 3 , and have $x$ parallel to $\mathbf{b}_{\mathrm{m}}$ and $z$ parallel to $\mathbf{c}_{\mathrm{h}}$. Components in the signal (5.4) have been calculated from the wavefunction inferred from the interpretation of resonant $\mathrm{x}$-ray Bragg diffraction, and we find,

$$
<Q_{\mathrm{zz}}>=0.78 \pm 0.08 \text {, and } \frac{1}{6}<Q_{\mathrm{xx}}-Q_{\mathrm{yy}}>=-0.17 \pm 0.05
$$

These values in (5.4) gives a difference signal with a sign that agrees with observation [13].

\section{Conclusion}

Successful interpretations of two x-ray experiments on $\mathrm{V}_{2} \mathrm{O}_{3}$ have been recorded. In both cases, the interpreted data was gathered on magnetically ordered $\mathrm{V}_{2} \mathrm{O}_{3}$ and the data relate to the spatially anisotropic distributions of $\mathrm{V}$ charge and orbital magnetization.

\section{Acknowledgements}

Dr. L. Paolasini has answered several enquiries about the execution of the diffraction experiments he and his colleagues have made on $\mathrm{V}_{2} \mathrm{O}_{3}$, and provided unpublished data to enhance our study. We are grateful to Dr. M. W. Long and Dr. U. Staub for continued interest in our work on the interpretation of resonant x-ray Bragg diffraction. One of us (S.W.L.) has benefitted from discussions with Dr. R. M. Moon and Professor G. A. Sawatzky. 


\section{Figure Captions}

\section{Fig. 1}

Positions of the eight vanadium ions in the monoclinic unit cell together with the configuration of their magnetic moments which lie in the $\mathbf{a}_{\mathrm{m}}-\mathbf{c}_{\mathrm{m}}$ plane of the diagram [3]. The monoclinic Bragg wavevector $(20 \overline{2})_{m}$ is parallel to the trigonal axis $\mathbf{c}_{\mathrm{h}}$, and $\mathbf{b}_{\mathrm{m}}$ is normal to the plane of the diagram and parallel to $\mathbf{a}_{\mathrm{h}}$.

\section{Fig. 2}

Data on Cr-doped $\mathrm{V}_{2} \mathrm{O}_{3}$ held at temperature $=0.55 \mathrm{~T}_{\mathrm{N}}=100 \mathrm{~K}$, and $\tau_{m}=(111)_{m}$ with $\theta=20.8^{\circ}$ [8]. The curves are a fit of calculated structure factors and data. An azimuthal-angle scan is rotation of the crystal by $\psi$ about $\tau_{m}$. The origin of the scan has the plane defined by $\tau_{m}$ and $(20 \overline{2})_{m}$ normal to the plane of scattering. 


\section{References}

1. D. B. McWhan, T. M. Rice, and J. P. Remeika, Phys. Rev. Lett. 23 (1969) 1334.

2. P. D. Dernier and M. Marezio, Phys. Rev. B2 (1970) 3771.

3. R. M. Moon, Phys. Rev. Lett. 25 (1970) 527.

4. D. B. McWhan et al., Phys. Rev. B7 (1973) 1920.

5. N. F. Mott, Metal-Insulator Transitions (Taylor and Francis, London, 1990).

6. T. M. Rice, Spectroscopy of Mott Insulators and Correlated Metals, edited by A. Fujimori and Y. Tokura (Springer, Berlin, 1995).

7. F. Mila et al., Phys. Rev. Lett. 85 (2000) 1714.

8. $\quad$ L. Paolasini et al., Phys. Rev. Lett. 82 (1999) 4719.

9. L. Paolasini et al., submitted to J. Elect. Spect. \& Related Phenomena (2001).

10. L. Paolasini, private communication.

11. D. H. Templeton and L. K. Templeton, Phys. Rev. B49 (1994) 14850.

12. D. H. Templeton, Acta Cryst. A54 (1998) 158.

13. J. - H. Park et al., Phys. Rev. B61 (2000) 11506.

14. S. W. Lovesey, J. Phys.: Condens. Matter 10 (1998) 2505.

15. W. Neubeck et al., Phys. Rev. B63 (2001) 134430; J. Igarashi and M. Takahasji, Phys. Rev. B63 (2001) 184430.

16. S. W. Lovesey and E. Balcar, J. Phys.: Condens. Matter 9 (1997) 8679.

17. S. W. Lovesey, K. S. Knight and E. Balcar, Phys. Rev. B64 (2001) 054405.

18. S. W. Lovesey, K. S. Knight and E. Balcar, submitted to Phys. Rev. B (2001).

19. S. W. Lovesey, K. S. Knight and D. S. Sivia, Second International Workshop on Electron Correlations and Materials Properties, ed. A. Gonis and N. Kioussis (Kluwer Academic/Plenum Publishers, 2001).

20. B. T. Thole et al., Phys. Rev. Lett. 68 (1992) 1943. 